\title{
Disease activity and lifestyle influence comorbidities and cardiovascular events in patients with acromegaly
}

\author{
Chiara Sardella', Daniele Cappellani', Claudio Urbani', Luca Manetti', \\ Giulia Marconcini ${ }^{1}$, Luca Tomisti ${ }^{1}$, Isabella Lupi ${ }^{1}$, Giuseppe Rossi ${ }^{2}$, Ilaria Scattina ${ }^{1}$, \\ Martina Lombardi', Vitantonio Di Bello ${ }^{3}$, Claudio Marcocci ${ }^{1}$, Enio Martino ${ }^{1}$ and \\ Fausto Bogazzi ${ }^{1}$ \\ ${ }^{1}$ Department of Clinical and Experimental Medicine, Section of Endocrinology, University of Pisa, Pisa, Italy, \\ ${ }^{2}$ Epidemiology and Biostatistics Unit, Institute of Clinical Physiology, National Research Council (C.N.R.), \\ Pisa, Italy, and ${ }^{3}$ Department of Pathology, University of Pisa, Pisa, Italy
}

Correspondence should be addressed to F Bogazzi Email

fausto.bogazzi@med.unipi.it

\begin{abstract}
Objective: The primary objective of this study is to identify the predictors of comorbidities and major adverse cardiovascular events (MACE) that can develop after diagnosis of acromegaly. The role of therapy for acromegaly in the event of such complications was also evaluated.

Design and methods: Retrospective cohort study was conducted on 200 consecutive acromegalic patients in a tertiary referral center. The following outcomes were evaluated: diabetes, hypertension and MACE. Each patient was included in the analysis of a specific outcome, unless they were affected when acromegaly was diagnosed, and further classified as follows: (i) in remission after adenomectomy (Hx), (ii) controlled by somatostatin analogues (SSA) (SSAc) or (iii) not controlled by SSA (SSAnc). Data were evaluated using Cox regression analysis.

Results: After diagnosis of acromegaly, diabetes occurred in $40.8 \%$ of patients. The SSAnc group had a three-fold higher risk of diabetes (HR: 3.32, $P=0.006$ ), whereas the SSAc group had a 1.4-fold higher risk of diabetes (HR: 1.43, $P=0.38$ ) compared with the $\mathrm{Hx}$ group. Hypertension occurred in $35.5 \%$ of patients, after diagnosis. The determinants of hypertension were age (HR: 1.06, $P=0.01$ ) and BMI (HR: 1.05, $P=0.01$ ). MACE occurred in $11.8 \%$ of patients, after diagnosis. Age (HR: 1.09, $P=0.005$ ) and smoking habit (HR: 5.95, $P=0.01$ ) were predictors of MACE. Conversely, therapy for acromegaly did not influence hypertension or MACE.

Conclusion: After diagnosis of acromegaly, control of the disease (irrespective of the type of treatment) and lifestyle are predictors of comorbidities and major adverse cardiovascular events.
\end{abstract}

\section{Introduction}

Acromegaly is characterized by excess of chronic GH and IGF1 and is associated with an increased morbidity and mortality. This is mainly due to cardiovascular and cerebrovascular diseases, which may be further aggravated by comorbidities such as hypertension, diabetes or dyslipidemia, all contributing to a high-risk cardiovascular profile in patients with acromegaly $(1,2)$.
() 2016 European Society of Endocrinology Printed in Great Britain
Overall, the prevalence of systemic complications with the diagnosis of acromegaly is higher than in the general population. In fact, the prevalence of hypertension is approximately two-fold higher in acromegalic patients than in the normal population ( $46 \%$ vs $25 \%$ ) (3), and the prevalence of diabetes ranges from 15 to $38 \%(4,5,6,7)$. In addition, increased levels of triglycerides have been 
reported in acromegaly, whereas hypercholesterolemia has occasionally been found (8). However, despite the unfavorable cardiovascular profile, contrasting data have been reported regarding the prevalence of coronary artery disease in acromegaly $(9,10,11)$.

The effects of surgical and medical therapies for acromegaly on systemic complications have been extensively studied $(12,13,14,15)$. Several studies have reported the beneficial effects of disease control on the management of metabolic and cardiovascular comorbidities already present at the diagnosis of acromegaly $(16,17)$.

However, acromegaly is a chronic syndrome lasting decades, and systemic complications may occur over the lifespan of patients. Little is known regarding the predictors of the comorbidities and cardiovascular acute events after the diagnosis of acromegaly.

The primary aim of this study was to identify the determinants of comorbidities and cardiovascular events that may occur after diagnosis of acromegaly. The potential effect of therapy on acromegaly in the event of such complications was also evaluated.

\section{Patients and methods}

This was a retrospective cohort study aiming at identifying the determinants of comorbidities and cardiovascular events, after the diagnosis of acromegaly. The Ethics Committee of the University of Pisa approved this study (study no. 3916), and all patients gave their written informed consent for the scientific use of their data.

\section{Patients}

We retrieved the clinical records of patients with acromegaly who had been referred to the endocrinology section of the Department of Clinical and Experimental Medicine at the University of Pisa from December 1974 to December 2014.

Totally, 200 acromegalic patients $(58.5 \%$ females; median (IQR) age: 46 (38-56) years) were included in the study.

\section{Study design}

It is our practice to submit all patients with acromegaly to a complete study of systemic complications of acromegaly at the diagnosis of disease and during the follow-up visits.
For the purposes of this study, the evaluated comorbidities and cardiovascular events (outcomes) were as follows: (i) diabetes mellitus, (ii) arterial hypertension and (iii) major adverse cardiovascular events (MACE).

Each patient was evaluated for the occurrence of a specific outcome, separately (Fig. 1). If an outcome was already present at the diagnosis of acromegaly, the patient was excluded from the analysis of that specific outcome. At the time of the occurrence of a specific outcome, the patient was classified as follows: (i) in remission after pituitary adenomectomy (HX), (ii) controlled by somatostatin analogue (SSA) therapy (SSAc) and (iii) not controlled by SSA therapy (SSAnc). If a specific outcome did not occur throughout the entire study period, the patient was classified with one of the three abovementioned groups, according to the acromegaly status at the time of the last follow-up visit.

For each outcome, the analysis was performed comparing the data of the three treatment groups, as illustrated in Fig. 1.

\section{Treatment groups}

The choice of therapy for acromegaly, for each patient, was based on clinical grounds, available treatments during that period and the willingness of the patient and was independent of the purpose of this study.

Pituitary adenomectomy was considered as the firstline therapy. Therapy with SSAs was started for various reasons: (i) patients for whom surgery was not feasible or contraindicated, (ii) patients that refused the surgical option and (iii) patients with active disease after surgery and/or radiotherapy. The treatment with SSAs was titrated every 6 months based on serum IGF1 concentrations.

Therapy with pegvisomant was started alone or in association with SSA therapy in patients with uncontrolled disease under somatostatin analogue therapy.

The Hx group included patients in surgical remission for at least 12 months before the onset of the complication or, if no complications have developed, the last time they were examined at our hospital.

The SSA groups included patients treated for at least 12 months with SSA therapy before the onset of the complication or in those cases in whom no complications had developed, the last visit at which they were seen. Those patients that had been controlled for at least 12 months with SSA therapy were grouped as SSAc; otherwise they were grouped as SSAnc.

The exclusion criteria were as follows: (i) patients treated for less than 12 months with any treatment 


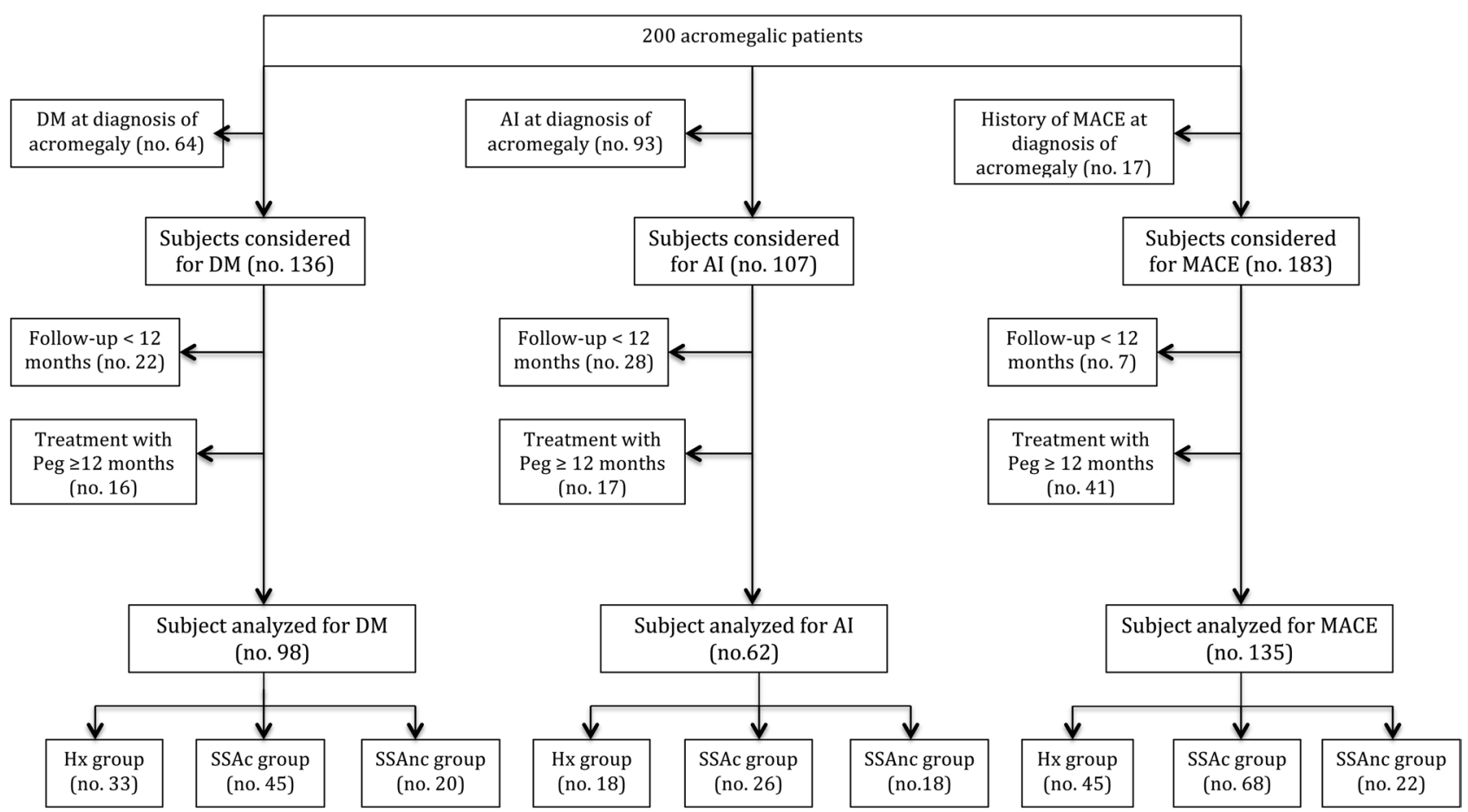

\section{Figure 1}

Study design. Two hundred acromegalic patients were retrospectively evaluated for the occurrence of the following complications (outcomes): diabetes mellitus (DM), arterial hypertension (AI) and major adverse cardiovascular events (MACE). Each patient was considered for the occurrence of a specific outcome, unless he/she was affected at the diagnosis of acromegaly. The Hx group included patients in surgical remission for at least 12 months before the onset of the complication or if no complications have developed, the last time they were examined at our hospital. The SSA groups included patients treated for at least 12 months with somatostatin analogue therapy (SSA) before the onset of the complication or in those cases in whom no complications had developed, the last visit at which they were seen. Those patients that had been controlled for at least 12 months with SSA therapy were grouped as SSAC, otherwise they were grouped as SSAnc. Patients were defined as in remission, uncontrolled or controlled for acromegaly based on the IGF1 index (ratio between the IGF1 concentration and the upper limit of the normal range for age). Remission or controlled acromegaly was defined when the IGF1 index was $\leq 1$. Patients were excluded from the analysis if (i) treated for less than 12 months with any treatment or (ii) treated for at least 12 months with pegvisomant (Peg) therapy before the onset of the complication or if no complications have developed, the last time they were examined. The analysis was carried out by comparing data among the three treatment groups.

and (ii) patients treated for at least 12 months with pegvisomant before the onset of the complication or, if no complications have developed, the last time they were examined (Fig. 1). The group of pegvisomant was not considered for statistical analysis because of the very small sample size and a much shorter follow-up period than that of patients treated with surgery and/or somatostatin analogue therapy.

\section{Diagnosis of acromegaly and endocrine evaluation}

The diagnosis of acromegaly was confirmed according to clinical and laboratory features, as reported $(18,19,20)$.
The estimated duration of acromegaly was expressed in months and consisted in the time interval between the onset of symptoms (assessed by the clinical history and comparison of previous personal photographs of face and hands over time, when available) and diagnosis of acromegaly.

Acromegaly was caused by a pituitary GH-secreting microadenoma in 63 cases $(31.5 \%)$ or by a macroadenoma in 137 cases $(68.5 \%)$.

At the diagnosis of acromegaly, partial anterior hypopituitarism was diagnosed in 44 subjects and treated accordingly: hypothyroidism $(n=9)$, hypogonadism $(n=28)$ and hypothyroidism combined with 
hypogonadism $(n=5)$ or with hypoadrenalism $(n=1)$. One patient had panhypopituitarism. After pituitary adenomectomy, hypopituitarism was recorded in 22 patients: hypothyroidism $(n=2)$, hypogonadism $(n=6)$ and GH deficiency $(n=1)$. The remaining eight patients had combined hypopituitarism and five patients had panhypopituitarism. Hormone replacement therapy was given as appropriate.

\section{Evaluation of comorbidities, cardiovascular events and their risk factors}

The diagnosis of comorbidities at baseline and during the study period was established according to the following most recent criteria: arterial hypertension was defined by the 2007 ESH-ESC guidelines (21), and diabetes mellitus was defined by the ADA 2014 guidelines (22).

In addition, we classified patients as hypertensives or diabetics if they were already receiving treatment for hypertension or diabetes respectively.

The major adverse cardiovascular events were defined as follows: (i) heart failure requiring hospitalization, (ii) myocardial infarction, (iii) arrhythmia requiring hospital admission and (iv) stroke including transient ischemic attack, as reported previously (23).

For all the outcomes, we considered the following risk factors: (i) age at diagnosis of acromegaly, (ii) sex, (iii) presence of hypopituitarism either at diagnosis of acromegaly or at the time of the occurrence of the outcome, (iv) body mass index either at diagnosis of acromegaly or at the time of the occurrence of the outcome and (v) biochemical status of acromegaly at the time of the occurrence of the outcome. In addition, family history
Table 1 Clinical and biochemical features of 200 patients at the diagnosis of acromegaly. Categorical data are expressed as $n(\%)$. Continuous data are expressed as median (interquartile range).

Sex: female
Pituitary macroadenoma
Hypopituitarism
Hypogonadism
Hypogonadism + hypothyroidism
Hypothyroidism
Hypothyroidism + hypoadrenalism
Panhypopituitarism
Age at diagnosis (years)
Estimated delay in diagnosis (months)
GH concentration at diagnosis ( $\mu$ g/L)
IGF1 index at diagnosis
Family history for diabetes
Family history for hypertension
Family history for hypercholesterolemia
Smokers at diagnosis
Former smokers at diagnosis
BMl at diagnosis (kg/m²)
Diabetes at diagnosis
Hypertension at diagnosis
Hypercholesterolemia at diagnosis
Major adverse cardiovascular events
at diagnosis

$117(58.5 \%)$

$137(68.5 \%)$

$44(22 \%)$

$28(14 \%)$

$5(2.5 \%)$

$9(4.5 \%)$

$1(0.5 \%)$

$1(0.5 \%)$

$46(38-56)$

$60(24-108)$

$10.6(4.92-24.35)$

$2.17(1.64-2.94)$

$55(27.5 \%)$

$92(46 \%)$

$12(6 \%)$

$48(26.08 \%)$

$41(22.28 \%)$

$27(24.67-31)$

$64(32 \%)$

$93(46.5 \%)$

$73(36.5 \%)$

$17(8.5 \%)$

BMI, body mass index.

for diabetes was considered in the analysis of diabetes. Family history for hypertension, smoking status, presence of diabetes and hypercholesterolemia, either at diagnosis of acromegaly or at the time of the occurrence of the outcome, was included for the analysis of hypertension. The presence of hypertension, hypercholesterolemia, diabetes either at diagnosis of acromegaly or at the time of the occurrence of the outcome and smoking status were evaluated for the MACE analysis.

Table 2 Distribution of risk factors of diabetes in the three treatment groups. Patients were defined as in remission, uncontrolled or controlled for acromegaly based on the IGF1 index (ratio between the IGF1 concentration and the upper limit of the normal range for age). Remission or controlled acromegaly was defined when the IGF1 index was $\leq 1$. Categorical data are expressed as $n(\%)$. Continuous data are expressed as median (interquartile range).

\section{Risk factor}

Females

Age at diagnosis (years)

Hypopituitarism at diagnosis

Hypopituitarism at outcome

Family history for diabetes

BMI at diagnosis $\left(\mathrm{kg} / \mathrm{m}^{2}\right)$

BMI at outcome $\left(\mathrm{kg} / \mathrm{m}^{2}\right)$

\begin{tabular}{c} 
Hx $n=33$ \\
\hline $19(57.58 \%)$ \\
$44(37.5-55)$ \\
$9(27.27 \%)$ \\
$16(48.48)$ \\
$8(27.59 \%)$ \\
$27(24-31.55)$ \\
$27.3(24.9-31.2)$ \\
\hline
\end{tabular}

\begin{tabular}{|c|c|}
\hline SSAnc $n=20$ & $P$ value \\
\hline $6(30.00 \%)$ & 0.05 \\
\hline $46.5(40.5-57)$ & 0.56 \\
\hline $8(40.00 \%)$ & 0.05 \\
\hline $9(45.0 \%)$ & 0.37 \\
\hline $3(16.67 \%)$ & 0.69 \\
\hline $29(25.3-31.45)$ & 0.78 \\
\hline $31(26.4-31.3)$ & 0.07 \\
\hline
\end{tabular}

BMI, body mass index; Hx: patients in remission after pituitary adenomectomy; SSAc: patients controlled with somatostatin analogue (SSA) therapy; SSAnc: patients uncontrolled with somatostatin analogue (SSA) therapy.

$P$ value $=$ comparison among the three groups. 


\section{Hormonal assay and acromegaly status}

Over the 40-year study period, the hormonal assays for GH and IGF1 changed, as expected. For the purposes of the study, disease activity was assessed by serum IGF1 concentrations. To compare serum IGF1 concentrations over the study period, we adopted the IGF1 index (the ratio between the IGF1 concentration and the upper limit of the normal range for age), as reported previously $(18,19,20)$. Remission or controlled disease was defined when the IGF1 index was $\leq 1$. However, uncontrolled disease was defined when the IGF1 index was $>1$.

\section{Statistical analysis}

Data were expressed as median and interquartile range for continuous variables and as frequency and percentage for categorical variables. The three treatment groups $(\mathrm{Hx}=$ patients in remission after pituitary adenomectomy; SSAC = patients controlled with somatostatin analogue SSA therapy; SSAnc=patients uncontrolled with somatostatin analogue SSA therapy) were compared by the non-parametric Kruskal-Wallis test for continuous variables and by the $\chi^{2}$ test for categorical variables. The relationship between risk factors and each outcome was evaluated by the Cox regression model in a univariate analysis. All significant or near significant risk factors in the univariate analysis were included in a multivariate Cox regression model. To evaluate the effect of treatments adjusted for significant risk factors, the treatment variable was always included in the multivariate Cox regression model.

The outcome-free survival was considered from the diagnosis of acromegaly to the occurrence of the outcome or to the last examination if a specific outcome did not occur throughout the entire study period.

Survival curves were estimated using the KaplanMeier method. The assumption of proportional hazards was addressed by scaled Schoenfeld residuals. A $P$ value of $<0.05$ was considered significant.

All computations were performed using the SPSS statistical package (SPSS).

\section{Results}

A total of 200 consecutive patients (117 women, 58.5\%) were included in the study; the baseline clinical and biochemical findings of the patients are summarized in Table 1. At the diagnosis of acromegaly, 64 (32\%) patients had diabetes mellitus and 93 (46.5\%) patients had arterial hypertension. In addition, 17 (8.5\%) patients already had a cardiovascular event, including two patients with ischemic stroke, two with heart failure, five with coronary heart disease and eight with arrhythmias. The median (IQR) follow-up time of the whole study group was 101 (49.5-169.5) months.
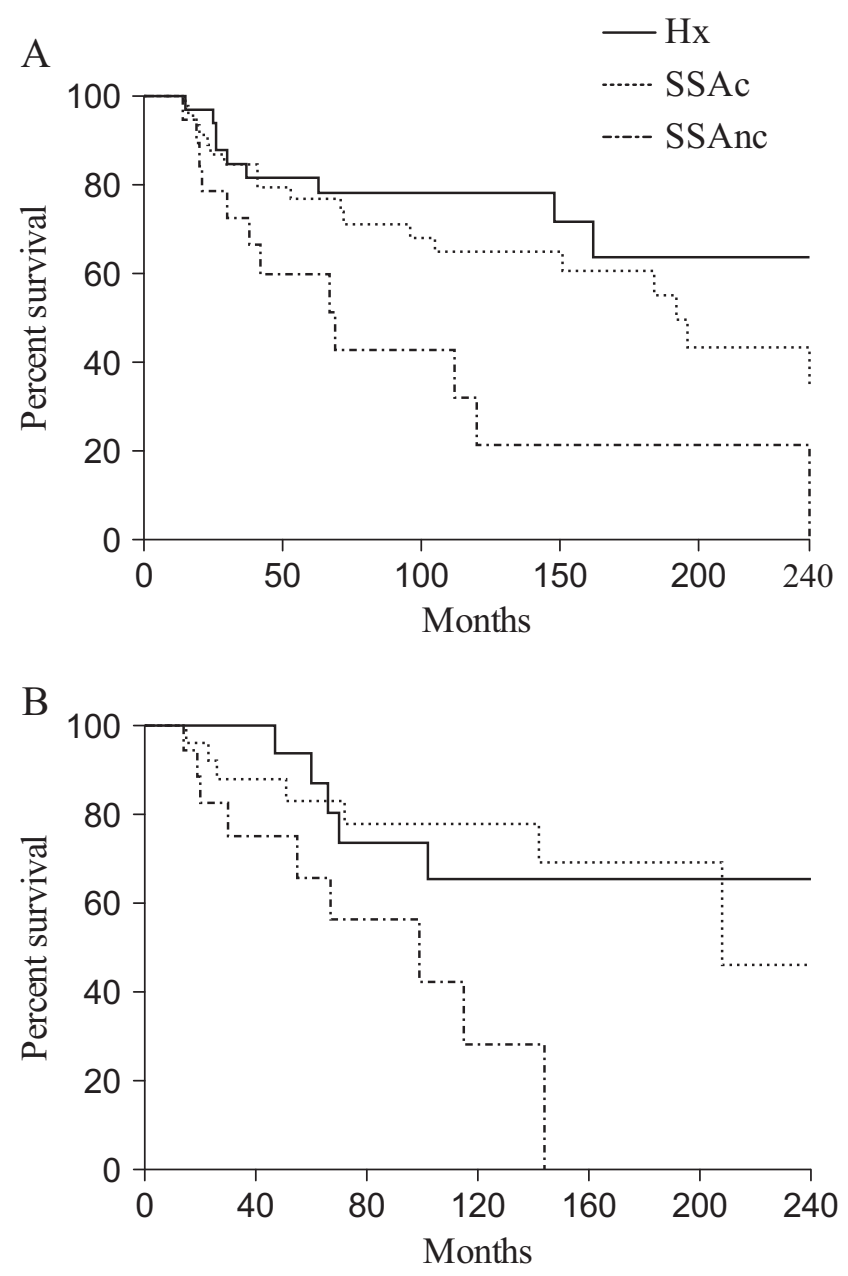

\section{Figure 2}

The Kaplan-Meier curves for the occurrence of diabetes mellitus (panel A) and arterial hypertension (panel B) in the three treatment groups over a 240-month period.

$\mathrm{Hx}=$ patients in remission after pituitary adenomectomy; SSAC = patients controlled with somatostatin analogue (SSA) therapy; SSAnc= patients uncontrolled with somatostatin analogue (SSA) therapy. Patients were defined as in remission, uncontrolled or controlled for acromegaly based on the IGF1 index (ratio between the IGF1 concentration and the upper limit of the normal range for age). Remission or controlled acromegaly was defined when the IGF1 index was $\leq 1$. 
Table 3 Risk factors of diabetes in univariate and multivariate Cox regression analyses. Patients were defined as uncontrolled or controlled for acromegaly based on the IGF1 index (ratio between the IGF1 concentration and the upper limit of the normal range for age). Controlled acromegaly was defined when the IGF1 index was $\leq 1$.

\begin{tabular}{l} 
Risk factor \\
\hline Age at diagnosis (years) \\
Sex (female) \\
Hypopituitarism at diagnosis \\
Hypopituitarism at outcome \\
Family history for diabetes \\
BMI at diagnosis $\left(\mathrm{kg} / \mathrm{m}^{2}\right)$ \\
BMI at outcome $\left(\mathrm{kg} / \mathrm{m}^{2}\right)$ \\
SSAC \\
SSAnc
\end{tabular}

\begin{tabular}{|c|c|}
\hline \multicolumn{2}{|c|}{ Univariate analysis } \\
\hline $\mathrm{HR}(95 \% \mathrm{Cl})$ & $P$ value \\
\hline $1.03(1.00-1.06)$ & 0.04 \\
\hline $0.96(0.52-1.75)$ & 0.90 \\
\hline $1.15(0.55-2.21)$ & 0.69 \\
\hline $0.66(0.34-1.21)$ & 0.18 \\
\hline $1.27(0.56-2.61)$ & 0.54 \\
\hline $1.01(0.91-1.11)$ & 0.85 \\
\hline $0.98(0.90-1.05)$ & 0.54 \\
\hline $1.47(0.67-3.43)$ & 0.34 \\
\hline $3.78(1.61-9.26)$ & 0.002 \\
\hline
\end{tabular}

\begin{tabular}{ccc}
\hline \multicolumn{2}{c}{ Multivariate analysis } \\
\hline HR $(95 \% \mathrm{Cl})$ & & $P$ value \\
\cline { 1 - 1 } $1.02(1.00-1.06)$ & & 0.08 \\
& \\
& \\
& \\
$1.43(0.65-3.34)$ & 0.38 \\
$3.32(1.41-8.20)$ & 0.006 \\
\hline
\end{tabular}

BMI, body mass index; HR, hazard ratio; 95\% Cl, 95\% confidence interval; SSAc: patients controlled with somatostatin analogue (SSA) therapy; SSAnc: patients uncontrolled with somatostatin analogue (SSA) therapy.

\section{Diabetes mellitus}

Overall, diabetes occurred in $40(40.8 \%)$ out of 98 patients after the diagnosis of acromegaly with a median (IQR) outcome-free survival of 72.5 (36-147.25) months. Sex and hypopituitarism were differentially distributed among the three treatment groups $(P=0.05$ and $P=0.05$ respectively, Table 2 ). In the univariate Cox analysis, the SSAnc group was associated with a nearly four-fold higher risk of diabetes than the Hx group, also adjusting for gender and hypopituitarism, (HR: 3.78, 95\% CI: 1.61-9.25, $P=0.002$; Fig. 2A and Table 3). Similarly, in the multivariate analysis, a lack of controlled acromegaly was the strongest determinant of the outcome (HR: 3.32, 95\% CI: 1.41-8.20, $P=0.006$ ). In addition, patients with controlled disease under SSA therapy showed a 1.4-fold higher risk of diabetes than those treated with surgery, although this difference was not statistically significant (HR: 1.43, 95\% CI: 0.65-3.34, $P=0.38$ ) (Table 3).

\section{Arterial hypertension}

Overall, hypertension occurred in $22(35.5 \%)$ out of 62 patients after the diagnosis of acromegaly with a median (IQR) outcome-free survival of 66 (34.5-120) months. As presented in Table 4, the three groups differed in terms of age at the diagnosis of acromegaly $(P=0.004)$ and of having diabetes when hypertension was diagnosed $(P=0.03)$. The univariate Cox analysis showed a six-fold higher risk of hypertension in patients with uncontrolled acromegaly (HR: 6.38, 95\%

Table 4 Distribution of the risk factors of hypertension in the three treatment groups. Patients were defined as in remission, uncontrolled or controlled for acromegaly based on the IGF1 index (ratio between the IGF1 concentration and the upper limit of the normal range for age). Remission or controlled acromegaly was defined when the IGF1 index was $\leq 1$. Categorical data are expressed as $n(\%)$. Continuous data are expressed as median (interquartile range).

\begin{tabular}{l}
\hline Risk factor \\
\hline Females \\
Age at diagnosis (years) \\
Hypopituitarism at diagnosis \\
Hypopituitarism at outcome \\
Family history for hypertension \\
Smokers at diagnosis \\
Former smokers at diagnosis \\
BMI at diagnosis $\left(\mathrm{kg} / \mathrm{m}^{2}\right)$ \\
BMI at outcome $\left(\mathrm{kg} / \mathrm{m}^{2}\right)$ \\
Diabetes at outcome \\
Hypercholesterolemia at outcome
\end{tabular}

\begin{tabular}{c}
\hline $\mathbf{H x} n=18$ \\
\hline $11(61.11 \%)$ \\
$39(28.5-44)$ \\
$4(22.22 \%)$ \\
$8(44.44 \%)$ \\
$3(18.75 \%)$ \\
$3(17.65 \%)$ \\
$3(17.65 \%)$ \\
$25.35(22.97-28.47)$ \\
$27(24.15-30.65)$ \\
$3(17.65 \%)$ \\
$9(52.94 \%)$ \\
\hline
\end{tabular}

\begin{tabular}{c}
\hline SSAC $n=26$ \\
\hline $14(53.85 \%)$ \\
$37(30-46.5)$ \\
$6(23.08 \%)$ \\
$12(46.15 \%)$ \\
$7(41.18 \%)$ \\
$8(40.0 \%)$ \\
$2(15.0 \%)$ \\
$24.75(23.1-28.46)$ \\
$25.6(23-29.55)$ \\
$10(38.46 \%)$ \\
$15(62.5 \%)$ \\
\hline
\end{tabular}

\begin{tabular}{|c|c|}
\hline SSAnc $n=18$ & $P$ value \\
\hline $6(33.33 \%)$ & 0.22 \\
\hline $48(42-57)$ & 0.004 \\
\hline $5(27.78 \%)$ & 0.91 \\
\hline $5(27.78 \%)$ & 0.43 \\
\hline $7(41.18 \%)$ & 0.29 \\
\hline $3(17.65 \%)$ & 0.08 \\
\hline $8(47.06 \%)$ & \\
\hline $25.3(24.5-30)$ & 0.54 \\
\hline $26(22.7-31)$ & 0.72 \\
\hline $11(61.11 \%)$ & 0.03 \\
\hline $10(55.56 \%)$ & 0.81 \\
\hline
\end{tabular}

BMI, body mass index; Hx: patients in remission after pituitary adenomectomy; SSAc: patients controlled with somatostatin analogue (SSA) therapy; SSAnc: patients uncontrolled with somatostatin analogue (SSA) therapy.

$P$ value = comparison among the three groups. 
Table 5 Risk factors of hypertension in univariate and multivariate Cox regression analyses. Patients were defined as uncontrolled or controlled for acromegaly based on the IGF1 index (ratio between the IGF1 concentration and the upper limit of the normal range for age). Controlled acromegaly was defined when the IGF1 index was $\leq 1$.

\begin{tabular}{|c|c|c|c|c|}
\hline \multirow[b]{2}{*}{ Risk factor } & \multicolumn{2}{|c|}{ Univariate analysis } & \multicolumn{2}{|c|}{ Multivariate analysis } \\
\hline & HR $(95 \% \mathrm{Cl})$ & $P$ value & HR $(95 \% \mathrm{Cl})$ & $P$ value \\
\hline Age at diagnosis (years) & $1.06(1.02-1.09)$ & 0.001 & $1.06(1.01-1.11)$ & 0.01 \\
\hline Sex (female) & $1.60(0.78-3.38)$ & 0.20 & & \\
\hline Hypopituitarism at diagnosis & $0.75(0.29-1.68)$ & 0.50 & & \\
\hline Hypopituitarism at outcome & $0.67(0.31-1.40)$ & 0.29 & & \\
\hline Family history for hypertension & $0.78(0.32-1.71)$ & 0.55 & & \\
\hline Smoker & $1.75(0.73-4.06)$ & 0.20 & & \\
\hline Former smoker & $1.91(0.71-4.82)$ & 0.19 & & \\
\hline Diabetes at diagnosis & $0.71(0.11-2.37)$ & 0.62 & & \\
\hline Diabetes at outcome & $1.12(0.51-2.32)$ & 0.77 & & \\
\hline Hypercholesterolemia at diagnosis & $1.24(0.51-2.71)$ & 0.61 & & \\
\hline Hypercholesterolemia at outcome & $1.08(0.52-2.31)$ & 0.84 & & \\
\hline BMI at diagnosis $\left(\mathrm{kg} / \mathrm{m}^{2}\right)$ & $1.11(0.97-1.27)$ & 0.12 & & \\
\hline BMI at outcome $\left(\mathrm{kg} / \mathrm{m}^{2}\right)$ & $1.09(1.0-1.19)$ & 0.05 & $1.05(0.95-1.16)$ & 0.01 \\
\hline SSAC & $1.49(0.54-4.75)$ & 0.45 & $0.67(0.19-2.25)$ & 0.51 \\
\hline SSAnc & $6.38(2.02-22.3)$ & 0.001 & $1.70(0.53-5.69)$ & 0.37 \\
\hline
\end{tabular}

$\mathrm{BMI}$, body mass index; $\mathrm{HR}$, hazard ratio; $95 \% \mathrm{Cl}, 95 \%$ confidence interval; SSAc: patients controlled with somatostatin analogue (SSA) therapy; SSAnc: patients uncontrolled with somatostatin analogue (SSA) therapy.

CI: $2.02-22.30, P=0.001)$ compared with patients in remission after surgery (Fig. $2 \mathrm{~B}$ and Table 5). However, age at the diagnosis of acromegaly (HR: 1.06, 95\% CI: $1.01-1.11, P=0.01)$ and body mass index at the occurrence of hypertension (HR: 1.05, 95\% CI: $0.95-1.16, P=0.01)$ were the predictors of the outcome in the multivariate analysis. Conversely, uncontrolled acromegaly at the outcome was not a predictor of hypertension (Table 5).

\section{MACE}

After diagnosis of acromegaly, MACE occurred in $16(11.8 \%)$ out of 135 patients with a median (IQR)

Table 6 Distribution of the risk factors for major adverse cardiovascular events (MACE) in the three treatment groups. Patients were defined as in remission, uncontrolled or controlled for acromegaly based on the IGF1 index (ratio between the IGF1 concentration and the upper limit of the normal range for age). Remission or controlled acromegaly was defined when the IGF1 index was $\leq 1$. Categorical data are expressed as $n(\%)$. Continuous data are expressed as median (interquartile range).

Risk factor
Females
Age at diagnosis (years)
Hypopituitarism at diagnosis
Hypopituitarism at outcome
BMI at diagnosis $\left(\mathrm{kg} / \mathrm{m}^{2}\right)$
BMI at outcome $\left(\mathrm{kg} / \mathrm{m}^{2}\right)$
Hypertension at diagnosis
Hypertension at outcome
Diabetes at diagnosis
Diabetes at outcome
Hypercholesterolemia at diagnosis
Hypercholesterolemia at outcome
Smokers
Former smokers

\begin{tabular}{c} 
Hx $n=45$ \\
\hline $27(60.0 \%)$ \\
$45(38.25-54)$ \\
$10(22.22 \%)$ \\
$16(35.56 \%)$ \\
$27.6(25.4-33.25)$ \\
$28.5(25.6-33.5)$ \\
$22(48.89 \%)$ \\
$33(73.33 \%)$ \\
$13(28.89 \%)$ \\
$19(42.22 \%)$ \\
$13(28.89 \%)$ \\
$26(57.78 \%)$ \\
$11(26.19 \%)$ \\
$9(21.43 \%)$ \\
\hline
\end{tabular}

\begin{tabular}{c}
\hline SSAC $n=68$ \\
\hline $44(64.71 \%)$ \\
$46(38.25-52)$ \\
$9(13.24 \%)$ \\
$21(30.88 \%)$ \\
$26.4(24-30.7)$ \\
$27.95(24.2-30.5)$ \\
$30(44.12 \%)$ \\
$48(71.64 \%)$ \\
$20(29.41 \%)$ \\
$41(61.19 \%)$ \\
$20(29.41 \%)$ \\
$44(65.67 \%)$ \\
$12(20.69 \%)$ \\
$10(17.24 \%)$ \\
\hline
\end{tabular}

\begin{tabular}{|c|c|}
\hline SSAnc $n=22$ & $P$ value \\
\hline $7(31.82 \%)$ & 0.02 \\
\hline $52(42-59)$ & 0.37 \\
\hline $6(27.27 \%)$ & 0.25 \\
\hline $6(27.27 \%)$ & 0.77 \\
\hline $28(25.15-31.02)$ & 0.37 \\
\hline $29.95(22.98-31)$ & 0.27 \\
\hline $12(54.55 \%)$ & 0.67 \\
\hline $13(59.09 \%)$ & 0.45 \\
\hline $11(50.0 \%)$ & 0.16 \\
\hline $15(68.18 \%)$ & 0.06 \\
\hline $7(31.82 \%)$ & 0.97 \\
\hline $15(68.18 \%)$ & 0.61 \\
\hline $3(14.29 \%)$ & 0.07 \\
\hline $10(47.62 \%)$ & \\
\hline
\end{tabular}

BMI, body mass index; Hx: patients in remission after pituitary adenomectomy; SSAc: patients controlled with somatostatin analogue (SSA) therapy; SSAnc: patients uncontrolled with somatostatin analogue (SSA) therapy.

$P$ value $=$ comparison among the three groups 
Table 7 Risk factors for major adverse cardiovascular events (MACE) in univariate and multivariate Cox regression analyses. Patients were defined as uncontrolled or controlled for acromegaly based on the IGF1 index (ratio between the IGF1 concentration and the upper limit of the normal range for age). Controlled acromegaly was defined when the IGF1 index was $\leq 1$.

\begin{tabular}{l}
\hline \\
\hline Age at diagnosis (years) \\
Sex (female) \\
Hypopituitarism at diagnosis \\
Hypopituitarism at outcome \\
Diabetes at diagnosis \\
Diabetes at outcome \\
Hypertension at diagnosis \\
Hypertension at outcome \\
Hypercholesterolemia at diagnosis \\
Hypercholesterolemia at outcome \\
Smokers \\
Former smokers \\
BMI at diagnosis $\left(\mathrm{kg} / \mathrm{m}^{2}\right)$ \\
BMI at outcome $\left(\mathrm{kg} / \mathrm{m}^{2}\right)$ \\
SSAc \\
SSAnc
\end{tabular}

\begin{tabular}{c}
\multicolumn{1}{c}{ Univariate } \\
\hline HR $(95 \% \mathrm{Cl})$ \\
\hline $1.07(1.02-1.11)$ \\
$1.16(0.45-2.82)$ \\
$0.37(0.06-1.28)$ \\
$0.66(0.34-1.21)$ \\
$2.57(1.04-6.14)$ \\
$1.20(0.49-2.99)$ \\
$3.33(1.38-8.83)$ \\
$2.76(0.78-17.49)$ \\
$1.55(0.61-3.74)$ \\
$1.99(0.72-6.96)$ \\
$2.08(0.54-6.93)$ \\
$4.00(1.37-11.49)$ \\
$1.05(0.95-1.16)$ \\
$1.04(0.96-1.12)$ \\
$0.66(0.22-2.06)$ \\
$2.04(0.42-7.92)$ \\
\hline
\end{tabular}

\begin{tabular}{cc}
\hline analyses & \\
\hline$P$ value \\
\hline 0.001 \\
0.74 \\
0.13 \\
0.18 \\
0.04 \\
0.68 \\
0.01 \\
0.13 \\
0.34 \\
0.19 \\
0.26 \\
0.01 \\
0.31 \\
0.34 \\
0.46 \\
0.34
\end{tabular}

\begin{tabular}{cc}
\hline \multicolumn{2}{c}{ Multivariate analyses } \\
\cline { 1 - 2 } HR $(95 \% \mathrm{Cl})$ & $\frac{P \text { value }}{0.005}$ \\
& \\
& \\
$0.89(1.02-1.17)$ & 0.86 \\
$1.33(0.41-4.66)$ & \\
& \\
& \\
& \\
$5.95(1.52-25.26)$ & 0.01 \\
$0.80(0.22-2.92)$ & 0.73 \\
$1.68(0.27-8.75)$ & 0.55 \\
\hline
\end{tabular}

BMI, body mass index; HR, hazard ratio; $95 \% \mathrm{Cl}, 95 \%$ confidence interval; SSAc: patients controlled with somatostatin analogue (SSA) therapy; SSAnc: patients uncontrolled with somatostatin analogue (SSA) therapy.

outcome-free survival of 83 (38-138) months. Of these, 6 patients had a myocardial infarction, 2 had an ischemic stroke, 6 had arrhythmias and 2 had heart failure. The distribution of the risk factors for MACE did not differ among the three groups (Table 6). In the multivariate Cox analysis, significant risk factors for the occurrence of MACE were age at the diagnosis of acromegaly (HR: 1.09, 95\% CI: 1.02-1.17, $P=0.005$ ) and smoking habit (HR: 5.95, 95\% CI: 1.52-25.26, P=0.01) (Table 7). Therapy for acromegaly did not influence the occurrence of the outcome. Likewise, diabetes mellitus and arterial hypertension were not associated with an increased risk of MACE (Table 7).

\section{Discussion}

Our results revealed that persistent acromegaly might be responsible for the late occurrence of diabetes mellitus. Conversely, the development of arterial hypertension and cardiovascular acute events seem to be dependent on factors that were not directly related to acromegaly.

Acromegaly is a chronic disease, which is associated with an increased morbidity and mortality along with the high risk of hospitalization and costs, mainly due to inadequate disease management and/or poor disease control (24). In fact, although the control of acromegaly can lead to a significant improvement in cardiovascular and metabolic comorbidities, a recent expert consensus document stated that 'comorbidities related to acromegaly should be managed as they are in the general population because they lead to increased mortality' (16). Thus, it is important to better understand what elements predispose acromegalic patients to develop metabolic and cardiovascular complications, as well as cardiovascular acute events, after acromegaly has been diagnosed.

Overall, systemic complications occurred in a significant proportion of patients in our study: after acromegaly had been diagnosed, diabetes was found in $41 \%$ and hypertension in 35\% of patients. Likewise, the occurrence of MACE during the study period (11.8\% of the patients) and the prevalence of MACE at baseline (8.5\% of the patients) were not negligible given the relatively young age of the study population. However, it is worth noting that the study population presented a high prevalence of cardiovascular risk factors; in addition, no case of cardiovascular death was reported during the follow-up period. This could be explained by considering that the timely treatment of diabetes, hypertension and hypopituitarism was carried out in the majority of acromegalic patients who had been referred to our center for the duration of their follow-up. In fact, it has been reported that the intensive treatment of multiple risk factors can reduce the risk of cardiovascular acute events by $50 \%$ in a population at high-risk cardiovascular profile, such as patients with type 2 diabetes (25). 
It has long been recognized that acromegaly is correlated with mortality and systemic complications $(18,26,27,28,29)$. A previous retrospective study evaluated the association of overall GH and IGF1 exposure with the occurrence of cardiovascular disease, cerebrovascular disease and diabetes in patients with treated acromegaly. Impaired glucose tolerance, diabetes and ischemic heart disease were associated with increased overall GH exposure, whereas cardiomyopathy and stroke were associated with increased overall IGF1 exposure (30).

However, whether there is an interplay between the disease activity and other factors in terms of systemic comorbidities and cardiovascular events still needs to be evaluated.

In this study, using Cox regression analysis, we investigated the role of several factors on the onset of diabetes, hypertension and MACE.

Our results showed that uncontrolled acromegaly was the strongest determinant of the development of diabetes (HR: 3.32 95\% CI: 1.41-8.2, P=0.006). This highlights the detrimental effect of not controlling the disease on glucose metabolism in the acromegalic population.

Age at the diagnosis of acromegaly significantly correlated with the development of hypertension (HR: $1.06,95 \% \mathrm{CI}: 1.01-1.11, P=0.01$ ) and the occurrence of MACE (HR: 1.09, 95\% CI: 1.02-1.17, $P=0.005$ ), whereas disease activity did not. In contrast, in a recent study, we observed a shift toward hypertension in normotensive patients with uncontrolled acromegaly after 24 months of SSA therapy. However, baseline blood glucose and serum insulin levels were higher in normotensive patients who developed hypertension compared with those who did not, suggesting an interplay between glucose metabolism and the development of hypertension in acromegalic patients (20).

A higher body mass index and smoking habit were also predictors of hypertension (HR: 1.05, 95\% CI: $0.95-1.16, P=0.01)$ and cardiovascular acute events (HR: 5.95, 95\% CI: 1.52-25.2, $P=0.01$ ), respectively, thus highlighting the importance of lifestyle interventions in acromegalic patients.

Taken together, these findings suggest that the lack of a tight control of acromegaly activity plays a central role in the development of metabolic complications, whereas older age at the diagnosis of the disease influences the development of cardiovascular complications and events. In addition, being overweight/obese and smoking habit increased cardiovascular risk in acromegalic patients as in the general population.
It has recently been reported how the effect of GH on body composition can lead to a specific pattern in the reduced storage of adipose tissue in central depots and a shift of excess lipid to intermuscular adipose tissue in acromegalic patients. These alterations in the adipose tissue metabolism can be associated with a metabolic risk and may be only partially reversed by surgery (31).

According to the results of multivariate Cox regression analyses, we found that once the control of acromegaly has been achieved, the choice of therapy (pituitary surgery or SSA) does not significantly influence the occurrence of diabetes after diagnosis of acromegaly. However, patients with controlled acromegaly under SSA therapy had a 1.5 -fold higher risk of diabetes than those cured by surgery, although this finding was not statistically significant. It has been reported that SSA can worsen glucose metabolism by inhibiting insulin secretion $(16,19)$ and may be less effective than pituitary surgery in reducing the risk of death in patients affected by diabetes mellitus (18). However, it is possible that the relatively small number of patients included in the study affected the power of the analysis.

Moreover, patients under therapy with pegvisomant were not included in the analysis due to the small numbers. However, this subset of patients may provide useful information because pegvisomant could be associated with a better glycemic profile $(2,19)$.

Further studies are thus needed to compare the effects of different treatments for acromegaly on the onset of systemic comorbidities, late after diagnosis of acromegaly.

The limitations of our study are mainly due to its observational nature. In addition, although the IGF1 concentrations at the occurrence of the outcome correlated strongly with mean IGF1 concentrations during the preceding follow-up period (18), as previously reported, it cannot be ruled out that the duration of the control or failure to control acromegaly before the onset of the outcome may have played a role.

In conclusion, the continuous control of acromegaly activity may be beneficial in reducing the risk of developing diabetes late after diagnosis of acromegaly.

In addition, lifestyle interventions such as control of body weight and avoiding smoking should be part of the whole management of the acromegalic patient in order to reduce cardiovascular diseases.

\section{Declaration of interest}

The authors declare that there is no conflict of interest that could be perceived as prejudicing the impartiality of the research reported. 
Funding

This work was partly supported by an unrestricted grant from Pfizer S.p.A. Italy.

\section{References}

1 Colao A, Ferone D, Marzullo P \& Lombardi G. Systemic complications of acromegaly: epidemiology, pathogenesis, and management. Endocrine Review 200425 102-152. (doi:10.1210/er.2002-0022)

2 Berg C, Petersenn S, Lahner H, Herrmann BL, Buchfelder M, Droste M, Stalla GK, Strasburger CJ, Roggenbuck U, Lehmann N et al. Cardiovascular risk factors in patients with uncontrolled and long-term acromegaly: comparison with matched data from the general population and the effect of disease control. Journal of Clinical Endocrinology and Metabolism 201095 3648-3656. (doi:10.1210/ jc.2009-2570)

3 Vitale G, Pivonello R, Auriemma RS, Guerra E, Milone F, Savastano S, Lombardi G \& Colao A. Hypertension in acromegaly and in the normal population: prevalence and determinants. Clinical Endocrinology 200563 470-476. (doi:10.1111/j.13652265.2005.02370.x)

4 Fieffe S, Morange I, Petrossians P, Chanson P, Rohmer V, Cortet C, Borson-Chazot F, Brue T \& Delemer B. Diabetes in acromegaly, prevalence, risk factors, and evolution: data from the French Acromegaly Registry. European Journal of Endocrinology 2011164 877-884. (doi:10.1530/EJE-10-1050)

5 Nabarro JD. Acromegaly. Clinical Endocrinology 198726 481-512. (doi:10.1111/j.1365-2265.1987.tb00805.x)

6 Ronchi C, Epaminonda P, Cappiello V, Beck-Peccoz P \& Arosio M. Effects of two different somatostatin analogs on glucose tolerance in acromegaly. Journal of Endocrinological Investigation 200225 502-507. (doi:10.1007/BF03345491)

7 Alexopoulou O, Bex M, Kamenicky P, Mvoula AB, Chanson P \& Maiter D. Prevalence and risk factors of impaired glucose tolerance and diabetes mellitus at diagnosis of acromegaly: a study in 148 patients. Pituitary 201417 81-89. (doi:10.1007/ s11102-013-0471-7)

8 Sesmilo G, Fairfield WP, Katznelson L, Pulaski K, Freda PU, Bonert V, Dimaraki E, Stavrou S, Vance ML, Hayden D et al. Cardiovascular risk factors in acromegaly before and after normalization of serum IGF-I levels with the GH antagonist pegvisomant. Journal of Clinical Endocrinology and Metabolism 200287 1692-1699. (doi:10.1210/ jcem.87.4.8364)

9 Mosca S, Paolillo S, Colao A, Bossone E, Cittadini A, Iudice FL, Parente A, Conte S, Rengo G, Leosco D et al. Cardiovascular involvement in patients affected by acromegaly: an appraisal. Internal Journal of Cardiology 2013167 1712-1718. (doi:10.1016/j. ijcard.2012.11.109)

10 Bogazzi F, Battolla L, Spinelli C, Rossi G, Gavioli S, Di Bello V, Cosci C, Sardella C, Volterrani D, Talini E et al. Risk factors for development of coronary heart disease in patients with acromegaly: a five-year prospective study. Journal of Clinical Endocrinology and Metabolism 200792 4271-4277. (doi:10.1210/jc.2007-1213)

11 Cannavo S, Almoto B, Cavalli G, Squadrito S, Romanello G, Vigo MT, Fiumara F, Benvenga S \& Trimarchi F. Acromegaly and coronary disease: an integrated evaluation of conventional coronary risk factors and coronary calcifications detected by computed tomography. Journal of Clinical Endocrinology and Metabolism 200691 3766-3772. (doi:10.1210/jc.2005-2857)

12 Annamalai AK, Webb A, Kandasamy N, Elkhawad M, Moir S, Khan F, Maki-Petaja K, Gayton EL, Strey CH, O'Toole S et al. A comprehensive study of clinical, biochemical, radiological, vascular, cardiac, and sleep parameters in an unselected cohort of patients with acromegaly undergoing presurgical somatostatin receptor ligand therapy.
Journal of Clinical Endocrinology and Metabolism 201398 1040-1050. (doi:10.1210/jc.2012-3072)

13 Reyes-Vidal C, Fernandez JC, Bruce JN, Crisman C, Conwell IM, Kostadinov J, Geer EB, Post KD \& Freda PU. Prospective study of surgical treatment of acromegaly: effects on ghrelin, weight, adiposity, and markers of CV risk. Journal of Clinical Endocrinology and Metabolism 201499 4124-4132. (doi:10.1210/jc.2014-2259)

14 Minniti G, Moroni C, Jaffrain-Rea ML, Esposito V, Santoro A, Affricano C, Cantore G, Tamburrano G \& Cassone R. Marked improvement in cardiovascular function after successful transsphenoidal surgery in acromegalic patients. Clinical Endocrinology 200155 307-313. (doi:10.1046/j.1365-2265.2001.01343.x)

15 Jaffrain-Rea ML, Minniti G, Moroni C, Esposito V, Ferretti E, Santoro A, Infusino T, Tamburrano G, Cantore G \& Cassone R. Impact of successful transsphenoidal surgery on cardiovascular risk factors in acromegaly. European Journal of Endocrinology 2003148 193-201. (doi:10.1530/eje.0.1480193)

16 Giustina A, Chanson P, Kleinberg D, Bronstein MD, Clemmons DR, Klibanski A, van der Lely AJ, Strasburger CJ, Lamberts SW, Ho KK et al. Expert consensus document: a consensus on the medical treatment of acromegaly. Nature Reviews Endocrinology 201410 243-248. (doi:10.1038/nrendo.2014.21)

17 Kuhn E, Maione L, Bouchachi A, Roziere M, Salenave S, BraillyTabard S, Young J, Kamenicky P, Assayag P \& Chanson P. Long-term effects of pegvisomant on comorbidities in patients with acromegaly: a retrospective single-center study. European Journal of Endocrinology 2015173 693-702. (doi:10.1530/EJE-15-0500)

18 Bogazzi F, Colao A, Rossi G, Lombardi M, Urbani C, Sardella C, Iannelli A, Scattina I, Manetti L, Del Sarto S et al. Comparison of the effects of primary somatostatin analogue therapy and pituitary adenomectomy on survival in patients with acromegaly: a retrospective cohort study. European Journal of Endocrinology 2013169 367-376. (doi:10.1530/EJE-13-0166)

19 Urbani C, Sardella C, Calevro A, Rossi G, Scattina I, Lombardi M, Lupi I, Manetti L, Martino E \& Bogazzi F. Effects of medical therapies for acromegaly on glucose metabolism. European Journal of Endocrinology 2013169 99-108. (doi:10.1530/EJE-13-0032)

20 Sardella C, Urbani C, Lombardi M, Nuzzo A, Manetti L, Lupi I, Rossi G, Del Sarto S, Scattina I, Di Bello V et al. The beneficial effect of acromegaly control on blood pressure values in normotensive patients. Clinical Endocrinology 201481 573-581. (doi:10.1111/cen.12455)

21 Mancia G, De Backer G, Dominiczak A, Cifkova R, Fagard R, Germano G, Grassi G, Heagerty AM, Kjeldsen SE, Laurent S et al. 2007 Guidelines for the management of arterial hypertension: the Task Force for the Management of Arterial Hypertension of the European Society of Hypertension (ESH) and of the European Society of Cardiology (ESC). Journal of Hypertension 200725 1105-1187. (doi:10.1097/HJH.0b013e3281fc975a)

22 American Diabetes Association. Standards of medical care in diabetes - 2014. Diabetes Care 201437 (Supplement 1) S14-S80. (doi:10.2337/dc14-S014)

23 Yiu KH, Jim MH, Siu CW, Lee CH, Yuen M, Mok M, Shea YF, Fan K, Tse HF \& Chow WH. Amiodarone-induced thyrotoxicosis is a predictor of adverse cardiovascular outcome. Journal of Clinical Endocrinology and Metabolism 200994 109-114. (doi:10.1210/jc.2008-1907)

24 Burton T, Le Nestour E, Bancroft T \& Neary M. Real-world comorbidities and treatment patterns of patients with acromegaly in two large US health plan databases. Pituitary 201316 354-362. (doi:10.1007/s11102-012-0432-6)

25 Gaede P, Vedel P, Larsen N, Jensen GV, Parving HH \& Pedersen O. Multifactorial intervention and cardiovascular disease in patients with type 2 diabetes. New England Journal of Medicine $2003 \mathbf{3 4 8}$ 383-393. (doi:10.1056/NEJMoa021778)

26 Mercado M, Gonzalez B, Vargas G, Ramirez C, de los Monteros AL, Sosa E, Jervis P, Roldan P, Mendoza V, Lopez-Felix B et al. Successful mortality reduction and control of comorbidities in patients with 
acromegaly followed at a highly specialized multidisciplinary clinic. Journal of Clinical Endocrinology and Metabolism 201499 4438-4446. (doi:10.1210/jc.2014-2670)

27 Colao A, Pivonello R, Grasso LF, Auriemma RS, Galdiero M, Savastano $S$ \& Lombardi G. Determinants of cardiac disease in newly diagnosed patients with acromegaly: results of a 10 year survey study. European Journal of Endocrinology 2011165 713-721. (doi:10.1530/EJE-11-0408)

28 Berg C, Petersenn S, Walensi M, Mohlenkamp S, Bauer M, Lehmann N, Roggenbuck U, Moebus S, Broecker-Preuss M, Sandalcioglu IE et al. Cardiac risk in patients with treatment naive, first-line medically controlled and first-line surgically cured acromegaly in comparison to matched data from the general population. Experimental and Clinical Endocrinology \& Diabetes 2013 121 125-132. (doi:10.1055/s-0032-1314811)
29 Vilar L, Naves LA, Costa SS, Abdalla LF, Coelho CE \& Casulari LA. Increase of classic and nonclassic cardiovascular risk factors in patients with acromegaly. Endocrine Practice 200713 363-372. (doi:10.4158/EP.13.4.363)

30 Jayasena CN, Comninos AN, Clarke H, Donaldson M, Meeran K \& Dhillo WS. The effects of long-term growth hormone and insulinlike growth factor- 1 exposure on the development of cardiovascular, cerebrovascular and metabolic co-morbidities in treated patients with acromegaly. Clinical Endocrinology 201175 220-225. (doi:10.1111/ j.1365-2265.2011.04019.x)

31 Reyes-Vidal CM, Mojahed H, Shen W, Jin Z, Arias-Mendoza F, Fernandez JC, Gallagher D, Bruce JN, Post KD \& Freda PU. Adipose tissue redistribution and ectopic lipid deposition in active acromegaly and effects of Surgical Treatment. Journal of Clinical Endocrinology and Metabolism 2015100 2946-2955. (doi:10.1210/jc.2015-1917)

Received 29 June 2016

Revised version received 10 August 2016

Accepted 15 August 2016 